\title{
Global well-posedness for a Smoluchowski equation coupled with Navier-Stokes equations in 2D.
}

\author{
P. Constantin \\ Department of Mathematics, The University of Chicago \\ 5734 S. University Avenue, Chicago, Il 60637 \\ email const@math.uchicago.edu \\ and \\ Nader Masmoudi \\ Courant Institute, New York University \\ 251 Mercer St, New York NY 10012 \\ email:masmoudi@cims.nyu.edu
}

August 15, 2018

\begin{abstract}
$\underline{\text { Abstract }}$
We prove global existence for a nonlinear Smoluchowski equation (a nonlinear FokkerPlanck equation) coupled with Navier-Stokes equations in $2 d$. The proof uses a deteriorating regularity estimate in the spirit of [5] (see also [1])

Key words Nonlinear Fokker-Planck equations, Navier-Stokes equations, Smoluchowski equation, micro-macro interactions.

AMS subject classification 35Q30, 82C31, 76A05.
\end{abstract}

\section{Introduction}

Systems coupling fluids and particles are of great interest in many branches of applied physics and chemistry. The equations attempt to describe the behavior of complex mixtures of particles and fluids, and as such, they present numerous challenges, simultaneously at three levels: at the level of their derivation, the level of their numerical simulation and that of their mathematical treatment. In this paper we concentrate solely on one aspect of the mathematical treatment, the regularity of solutions. The particles in the system are described by a probability distribution $f(t, x, m)$ that depends on time $t$, macroscopic variable $x \in \mathbb{R}^{n}$, and particle configuration $m \in M$. Here $M$ is a smooth compact Riemannian manifold without boundary. The particles are transported by a fluid, agitated by thermal noise, and interact among themselves. This is reflected in a kinetic equation for the evolution of the probability distribution of the particles $([2,8])$. The interaction between particles - a micro-micro interaction - is modeled in a mean-field fashion by a potential that represents the tendency of particles to favor certain coherent configurations. The interaction between particles occurs only when the concentration of particles is sufficiently high. Mathematically, this term is responsible for the nonlinearity of the Smoluchowski (Fokker-Planck) equation, and physically, it is responsible 
for nematic phase transitions. Because the particles are considerably small, and for smooth flows, the Lagrangian transport of the particles is modeled using a Taylor expansion of the velocity field. This gives rise to a drift term in the Smoluchowski equation that depends on the spatial gradient of velocity. It is a macro-micro term, and it causes mathematical difficulties in the regularity theory.

The fluid is described by the incompressible Navier-Stokes equations. The microscopic particles add stresses to the fluid. This is the micro-macro interaction and it is the most puzzling and important physical aspect of the problem. Indeed, while a macro-micro interaction can be derived, in principle, by assuming that the macroscopic entities vary little on the scale of the microscopic ones, the "scaling up" of the effect of microscopic quantities to the macroscopic level is more mysterious. A principle based on an energy dissipation balance, and that recovers familiar results in simple cases was proposed in [6], where the regularity of nonlinear Fokker-Planck systems coupled with Stokes equations in 3D was also proved. The linear Fokker-Planck system coupled with Stokes equations was considered in [19]. The nonlinear Fokker-Planck equation driven by a time averaged Navier-Stokes system in 2D was studied in [7].

An approximate closure of the linear Fokker-Planck equation reduces the description to closed viscoelastic equations for the added stresses themselves. This leads to well-known nonNewtonian fluid models that have been studied extensively. For regularity results we refer to Lions and Masmoudi [18] where the existence of global weak solutions was proved for an Oldroyd-type model. In Guillopé and Saut [13] and [14], the existence of local strong solution was proved. Also, Fernández-Cara, Guillén and Ortega [11], [10] and [12] proved local well posedness in Sobolev spaces. We also mention Lin, Liu and Zhang [16] where a formulation based on the deformation tensor is used to study the Oldroyd-B model.

An other model for the polymers is the FENE dumbbell model. From mathematical point of view, this model was studied by several authors. In particular W. E, Li and Zhang [9], Jourdain, Lelievre and Le Bris [15] and Zhang and Zhang [20] proved local well-posedness. Moreover, Lin, Liu and Zhang [17] proved global existence near equilibrium.

\section{$1.1 \quad$ The model}

Consider the system

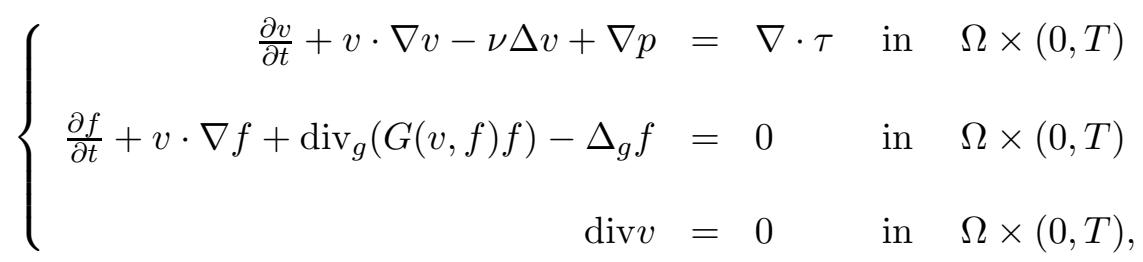

where $\tau_{i j}=\int_{M} \gamma_{i j}^{(1)}(m) f(t, x, m) d m+\int_{M} \int_{M} \gamma_{i j}^{(2)}\left(m_{1}, m_{2}\right) f\left(t, x, m_{1}\right) f\left(t, x, m_{2}\right) d m$. We denote $G(v, f)=\nabla_{g} U+W$ where $W=c_{\alpha}^{i j} \partial_{j} v_{i}$ and $U=K f$ is a potential given by

$$
U(t, x, m)=\int_{M} K(m, q) f(t, x, q) d q
$$

with a kernel $K$ which is a smooth, time and space independent symmetric function $K$ : $M \times M \rightarrow \mathbb{R}$. We also take $\Omega=\mathbb{R}^{2}$. 


\subsection{Statement of the result}

Theorem 1.1 Take $v(0) \in W^{1+\varepsilon_{0}, r} \cap L^{2}\left(\mathbb{R}^{2}\right)$ and $f(0) \in W^{1, r}\left(H^{-s}\right)$, for some $r>2$ and $\varepsilon_{0}>$ 0 and $f \geq 0, \int_{M} f_{0} \in L^{1} \cap L^{\infty}$. Then (1) has a global solution in $v \in L_{l o c}^{\infty}\left(W^{1, r}\right) \cap L_{l o c}^{2}\left(W^{2, r}\right)$ and $f \in L_{l o c}^{\infty}\left(W^{1, r}\left(H^{-s}\right)\right)$. Moreover, for $T>T_{0}>0$, we have $v \in L^{\infty}\left(\left(T_{0}, T\right) ; W^{2-\varepsilon, r}\right)$.

\subsection{Preliminaries}

We define $\mathcal{C}$ to be the ring of center 0 , of small radius $1 / 2$ and great radius 2 . There exist two nonnegative radial functions $\chi$ and $\varphi$ belonging respectively to $\mathcal{D}(B(0,1))$ and to $\mathcal{D}(\mathcal{C})$ so that

$$
\begin{gathered}
\chi(\xi)+\sum_{q \geq 0} \varphi\left(2^{-q} \xi\right)=1, \\
|p-q| \geq 2 \Rightarrow \operatorname{Supp} \varphi\left(2^{-\mathrm{q}} \cdot\right) \cap \operatorname{Supp} \varphi\left(2^{-\mathrm{p}} \cdot\right)=\emptyset .
\end{gathered}
$$

For instance, one can take $\chi \in \mathcal{D}(B(0,1))$ such that $\chi \equiv 1$ on $B(0,1 / 2)$ and take

$$
\varphi(\xi)=\chi(\xi / 2)-\chi(\xi) .
$$

Then, we are able to define the Littlewood-Paley decomposition. Let us denote by $\mathcal{F}$ the Fourier transform on $\mathbb{R}^{d}$. Let $h, \widetilde{h}, \Delta_{q}, S_{q}(q \in \mathbb{Z})$ be defined as follows:

$$
\begin{gathered}
h=\mathcal{F}^{-1} \varphi \text { and } \widetilde{h}=\mathcal{F}^{-1} \chi, \\
\Delta_{q} u=\mathcal{F}^{-1}\left(\varphi\left(2^{-q} \xi\right) \mathcal{F} u\right)=2^{q d} \int h\left(2^{q} y\right) u(x-y) d y, \\
S_{q} u=\mathcal{F}^{-1}\left(\chi\left(2^{-q} \xi\right) \mathcal{F} u\right)=2^{q d} \int \widetilde{h}\left(2^{q} y\right) u(x-y) d y .
\end{gathered}
$$

We use the para-product decomposition of Bony ([3])

$$
u v=T_{u} v+T_{v} u+R(u, v)
$$

where

$$
T_{u} v=\sum_{q \in \mathbb{Z}} S_{q-1} u \Delta_{q} v \quad \text { and } \quad R(u, v)=\sum_{\left|q-q^{\prime}\right| \leq 1} \Delta_{q^{\prime}} u \Delta_{q} v .
$$

We define the inhomogeneous and homogeneous Besov spaces by

Definition 1.2 Let $s$ be a real number, $p$ and $r$ two real numbers greater than 1 . Then we define the following norm

$$
\|u\|_{\widetilde{B}_{p, r}^{s}} \stackrel{\text { def }}{=}\left\|S_{0} u\right\|_{L^{p}}+\|\left(2^{q s}\left\|\Delta_{q} u\right\|_{\left.L^{p}\right)_{q \in \mathbb{N}}} \|_{\ell^{r}(\mathbb{N})}\right.
$$

and the following semi-norm

$$
\|u\|_{B_{p, r}^{s}} \stackrel{\text { def }}{=}\left\|\left(2^{q s}\left\|\Delta_{q} u\right\|_{L^{p}}\right)_{q \in \mathbb{Z}}\right\|_{\ell^{r}(\mathbb{Z})} .
$$

\section{Definition 1.3}

- Let $s$ be a real number, $p$ and $r$ two real numbers greater than 1 . We denote by $\widetilde{B}_{p, r}^{s}$ the space of tempered distributions $u$ such that $\|u\|_{\widetilde{B}_{p, r}^{s}}$ is finite. 
- If $s<d / p$ or $s=d / p$ and $r=1$ we define the homogeneous Besov space $B_{p, r}^{s}$ as the closure of compactly supported smooth functions for the norm $\|\cdot\|_{B_{p, r}^{s}}$.

We refer to [4] for the proof of the following results and for the multiplication law in Besov spaces.

\section{Lemma 1.4}

$$
\begin{gathered}
\left\|\Delta_{q} u\right\|_{L^{b}} \leq 2^{d\left(\frac{1}{a}-\frac{1}{b}\right) q}\left\|\Delta_{q} u\right\|_{L^{a}} \quad \text { for } b \geq a \geq 1 \\
\left\|e^{t \Delta} \Delta_{q} u\right\|_{L^{b}} \leq C 2^{-c t 2^{2 q}}\left\|\Delta_{q} u\right\|_{L^{b}}
\end{gathered}
$$

The following corollary is straightforward.

Corollary 1.5 If $b \geq a \geq 1$, then, we have the following continuous embeddings

$$
B_{a, r}^{s} \subset B_{b, r}^{s-d}\left(\frac{1}{a}-\frac{1}{b}\right) .
$$

Definition 1.6 Let $p$ be in $[1, \infty]$ and $r$ in $\mathbb{R}$; the space $\widetilde{L}_{T}^{p}\left(C^{r}\right)$ is the space of distributions $u$ such that

$$
\|u\|_{\widetilde{L}^{p}\left(0, T ; C^{r}\right)} \stackrel{\text { def }}{=} \sup _{q} 2^{q r}\left\|\Delta_{q} u\right\|_{L_{T}^{p}\left(L^{\infty}\right)}<\infty
$$

We will use the following theorem from [5]

Theorem 1.7 Let $v$ be the solution in $L_{T}^{2}\left(H^{1}\right)$ of the two dimensional Navier-Stokes system

$$
\left(N S_{\nu}\right)\left\{\begin{aligned}
\frac{\partial v}{\partial t}+v \cdot \nabla v-\nu \Delta v & =-\nabla p+f \\
\operatorname{div} v & =0 \\
\left.v\right|_{t=0} & =v_{0} .
\end{aligned}\right.
$$

with an initial data in $L^{2}$ and an external force $f$ in $L_{T}^{1}\left(C^{-1}\right) \cap L_{T}^{2}\left(H^{-1}\right)$; then, for any $\varepsilon$, a $T_{0}$ in the interval $] 0, T[$ exists such that

$$
\|\nabla v\|_{\widetilde{L}_{\left[T_{0}, T\right]}^{1}\left(C^{0}\right)} \leq \varepsilon
$$

\section{A deteriorating regularity estimate}

The main part of this section is the proof of a deteriorating regularity estimate for transport equations in the spirit of [1] and [5]. After this proof, we will apply this estimate in order to prove Theorem 1.1.

We also denote $H=\left(-\Delta_{g}+I\right)^{-s / 2}$ with $s>d / 2+1$.

Theorem 2.1 Let $\sigma$ and $\beta$ be two elements of $] 0,1[$ such that $\sigma+\beta<1$. A constant $C$ exists that satisfies the following properties. Let $T$ and $\lambda$ be two positive numbers and $v$ a smooth divergence free vector field so that

$$
\sigma-\lambda\|\nabla v\|_{\widetilde{L}_{T}^{1}\left(C^{0}\right)} \geq \beta \quad \text { and } \quad \sigma+\lambda\|\nabla v\|_{\widetilde{L}_{T}^{1}\left(C^{0}\right)} \leq 1-\beta .
$$

Consider two smooth functions $f$ and $v$ so that $f$ is the solution of

$$
\left\{\begin{aligned}
\partial_{t} f+v \cdot \nabla f+\operatorname{div}_{g}(G(v, f) f)-\Delta_{g} f & =0 \\
f_{\mid t=0} & =f_{0} .
\end{aligned}\right.
$$


Then we have, if $\lambda \geq 3 C$,

$$
M_{\lambda}^{\sigma}(f) \leq 3\left\|f_{0}\right\|_{B_{p, \infty}^{\sigma}\left(H^{-s}\right)}+\frac{3 C}{\lambda} M_{\lambda}^{\sigma+1}(v)
$$

where

$$
\begin{aligned}
M_{\lambda}^{\sigma}(v) & \stackrel{\text { def }}{=} \sup _{t \in[0, T], q} 2^{q \sigma-\Phi_{q, \lambda}(t)}\left\|\Delta_{q} v(t)\right\|_{L^{p}} \quad \text { or } \\
M_{\lambda}^{\sigma}(f) & \stackrel{\text { def }}{=} \sup _{t \in[0, T], q} 2^{q \sigma-\Phi_{q, \lambda}(t)}\left\|\Delta_{q} f(t)\right\|_{L^{p}\left(H^{-s}\right)} \quad \text { with } \\
\Phi_{q, \lambda}\left(t, t^{\prime}\right) & \stackrel{\text { def }}{=} \lambda \int_{t^{\prime}}^{t}\left(\left\|S_{q-1} \nabla v\left(t^{\prime \prime}\right)\right\|_{L^{\infty}}+1\right) d t^{\prime \prime}, \Phi_{q, \lambda}(t)=\Phi_{q, \lambda}(t, 0) .
\end{aligned}
$$

We will use the notation $f_{q} \stackrel{\text { def }}{=} \Delta_{q} f$. Applying the operator $\Delta_{q}$ to the transport equation (6), we get

$$
\left\{\begin{array}{r}
\partial_{t} f_{q}+S_{q-1} v \cdot \nabla f_{q}+\operatorname{div}_{g}\left(G\left(S_{q-1} v, S_{q-1} f\right) f_{q}\right)-\Delta_{g} f_{q}+R_{q}(v, f)=0 \\
f_{q \mid t=0}=\Delta_{q} f_{0}
\end{array}\right.
$$

where $R_{q}$ is a rest term.

We denote

$$
N_{q}^{2}(t, x)=\int_{M}\left|H f_{q}\right|^{2} d m
$$

Applying $H$ to (11) and taking the $L^{2}$ norm on $M$, we get

$$
\partial_{t} N_{q}^{2}+S_{q-1} v \cdot \nabla N_{q}^{2}+V\left(S_{q-1} v, S_{q-1} f, f_{q}\right)+\left|\nabla_{g} H f_{q}\right|^{2}+\int_{M} H f_{q}\left(H R_{q}(v, f)\right) d m=0
$$

where

$$
V(v, h, f)=\partial_{j} v_{i} \int_{M}\left(H \operatorname{div}_{g}\left(c_{\alpha}^{i j} f\right)\right)(H f) d m .+\int_{M}\left(H \operatorname{div}_{g}\left(\nabla_{g} h f\right)\right)(H f) d m .
$$

Hence, arguing as in [7], we have $\left|V\left(S_{q-1} v, f_{q}\right)\right| \leq C\left(\left|\nabla S_{q-1} v\right|+\left\|S_{q-1} f\right\|_{L^{2}(M)}\right) N_{q}^{2}$.

We will use now the following lemma, postponing its proof:

Lemma $2.2 R_{q}(v, f)$ satisfies

$$
\begin{aligned}
2^{q \sigma-\Phi_{q, \lambda}(t)} \| & H R_{q}(v(t), f(t)) \|_{L^{p}\left(L^{2}\right)} \leq C e^{C \lambda\|\nabla v\|_{\tilde{L}_{T}^{1}\left(C^{0}\right)}} \\
& \times\left(M_{\lambda}^{\sigma+1}(v)+\left(1+\left\|S_{q} \nabla v(t)\right\|_{L^{\infty}}+\sum_{\left|q^{\prime}-q\right| \leq N}\left\|\Delta_{q^{\prime}} \nabla v(t)\right\|_{L^{\infty}}\right) M_{\lambda}^{\sigma}(f)\right) .
\end{aligned}
$$

Taking the $L^{p}$ norm of $N_{q}$, we get

$$
\left\|N_{q}(t)\right\|_{L^{p}} \leq\left\|N_{q}(0)\right\|_{L^{p}}+\int_{0}^{t}\left\|H R_{q}\left(v\left(t^{\prime}\right), f\left(t^{\prime}\right)\right)\right\|_{L^{p}\left(L^{2}\right)}+\left\|\nabla S_{q} v\left(t^{\prime}\right)\right\|_{L^{\infty}}\left\|N_{q}\left(t^{\prime}\right)\right\|_{L^{p}} d t^{\prime} .
$$

After multiplication by $2^{q \sigma-\Phi_{q, \lambda}(t)}$, we get 


$$
\begin{aligned}
2^{q \sigma-\Phi_{q, \lambda}(t)}\left\|N_{q}(t)\right\|_{L^{p}} \leq 2^{q \sigma}\left\|N_{q}(0)\right\|_{L^{p}} & +\int_{0}^{t} 2^{-\Phi_{q, \lambda}\left(t, t^{\prime}\right)} 2^{q \sigma-\Phi_{q, \lambda}\left(t^{\prime}\right)}\left\|\nabla S_{q} v\left(t^{\prime}\right)\right\|_{L^{\infty}}\left\|N_{q}\right\|_{L^{p}} d t^{\prime} \\
& +\int_{0}^{t} 2^{-\Phi_{q, \lambda}\left(t, t^{\prime}\right)} 2^{q \sigma-\Phi_{q, \lambda}\left(t^{\prime}\right)}\left\|H R_{q}\left(v\left(t^{\prime}\right), f\left(t^{\prime}\right)\right)\right\|_{L^{p}\left(L^{2}\right)} d t^{\prime} .
\end{aligned}
$$

Then, using the inequality (15) and taking the sup over $q$, we get

$$
\begin{array}{r}
M_{\lambda}^{\sigma}(f) \leq\left\|f_{0}\right\|_{B_{p, \infty}^{\sigma}\left(H^{-s}\right)}+e^{C \lambda\|\nabla v\|_{\widetilde{L}_{T}^{1}\left(C^{0}\right)}} \sup _{t \in[0, T], q} \int_{0}^{t} 2^{-\Phi_{q, \lambda}\left(t, t^{\prime}\right)} \\
\times\left(M_{\lambda}^{\sigma+1}(v)+M_{\lambda}^{\sigma}(f)\left(1+2\left\|S_{q} \nabla v\left(t^{\prime}\right)\right\|_{L^{\infty}}+\sum_{\left|q^{\prime}-q\right| \leq N}\left\|\Delta_{q^{\prime}} \nabla v\left(t^{\prime}\right)\right\|_{L^{\infty}}\right)\right) d t^{\prime} .
\end{array}
$$

As $\lambda\|\nabla v\|_{\widetilde{L}_{T}^{1}\left(C^{0}\right)}$ is smaller than $(\sigma-\beta)$, we have

$$
e^{C \lambda\|\nabla v\|_{\tilde{L}_{T}^{1}\left(C^{0}\right)}} \leq e^{C(\sigma-\beta)} .
$$

Moreover, by definition of $\Phi_{q, \lambda}\left(t, t^{\prime}\right)$, it is obvious that

$$
\int_{0}^{t} 2^{-\Phi_{q, \lambda}\left(t, t^{\prime}\right)}\left(\left\|S_{q} \nabla v\left(t^{\prime}\right)\right\|_{L^{\infty}}+1\right) d t^{\prime} \leq \frac{1}{\lambda \log 2} .
$$

Then, we obtain that

$$
\begin{aligned}
M_{\lambda}^{\sigma}(f) & \leq\left\|f_{0}\right\|_{B_{p, \infty}^{\sigma}\left(H^{-s}\right)}+\frac{C}{\lambda} M_{\lambda}^{\sigma+1}(v)+C\|\nabla v\|_{\widetilde{L}_{T}^{1}\left(C^{0}\right)} M_{\lambda}^{\sigma}(f)+\frac{C}{\lambda} M_{\lambda}^{\sigma}(f) \\
& \leq\left\|f_{0}\right\|_{B_{p, \infty}^{\sigma}\left(H^{-s}\right)}+\frac{C}{\lambda} M_{\lambda}^{\sigma+1}(v)+\frac{2 C}{\lambda} M_{\lambda}^{\sigma}(f) .
\end{aligned}
$$

This proves the theorem of course if we prove the estimate (15) of the lemma. First of all, let 
us decompose the operator $R_{q}$. We have

$$
\begin{aligned}
R_{q}(v, f) & =\sum_{\ell=1}^{6} R_{q}^{\ell}(v, f) \quad \text { with } \\
R_{q}^{1}(v, f) & =\sum_{j=1}^{d} \Delta_{q}\left(T_{\partial_{j} f} v^{j}\right), \\
R_{q}^{2}(v, f) & =\sum_{j=1}^{d}\left[\Delta_{q}, T_{v^{j}} \partial_{j}\right] f, \\
R_{q}^{3}(v, f) & =\sum_{j=1}^{d} \Delta_{q} \partial_{j} R\left(v^{j}, f\right)+\Delta_{q-1} v^{j} \partial_{j} \Delta_{q+1} f_{q}-\Delta_{q-2} v^{j} \partial_{j} \Delta_{q-1} f_{q} \\
R_{q}^{4}(v, f) & =\sum_{i, j=1}^{d} \operatorname{div}_{g}\left(c_{\alpha}^{i j} \Delta_{q}\left(T_{f} \partial_{j} v^{i}\right)\right)+\operatorname{div}_{g}\left(\Delta_{q}\left(T_{f} \nabla_{g} U\right)\right), \\
R_{q}^{5}(v, f) & =\sum_{i, j=1}^{d} \operatorname{div}_{g}\left(c_{\alpha}^{i j}\left[\Delta_{q}, T_{\partial_{j} v^{i}}\right] f\right)+\operatorname{div}_{g}\left(\left[\Delta_{q}, T_{\nabla_{g} U}\right] f\right) \\
R_{q}^{6}(v, f) & =\sum_{i, j=1}^{d} \operatorname{div}_{g}\left(c_{\alpha}^{i j}\left(R\left(\partial_{j} v^{i}, f\right)+\Delta_{q-1} \partial_{j} v^{i} \Delta_{q+1} f_{q}-\Delta_{q-2} \partial_{i} v^{j} \Delta_{q-1} f_{q}\right)\right) \\
& +\sum_{i, j=1}^{d} \operatorname{div}_{g}\left(R\left(\nabla_{g} U, f\right)+\Delta_{q-1} \nabla_{g} U \Delta_{q+1} f_{q}-\Delta_{q-2} \nabla_{g} U \Delta_{q-1} f_{q}\right)
\end{aligned}
$$

Indeed,

$$
\begin{aligned}
\Delta_{q}(v \cdot \nabla f) & =\Delta_{q}\left(\sum_{j=1}^{d} T_{\partial_{j} f} v^{j}+T_{v^{j}} \partial_{j} f+R\left(v^{j}, \partial_{j} f\right)\right) \\
& =\sum_{\ell=1}^{2} R_{q}^{\ell}(v, f)+\sum_{j=1}^{d} T_{v^{j}} \partial_{j} \Delta_{q} f+\Delta_{q} R\left(v^{j}, \partial_{j} f\right),
\end{aligned}
$$

Then, we use that

$$
\begin{aligned}
\sum_{j=1}^{d} T_{v^{j}} \partial_{j} f_{q} & =\sum_{\left|q-q^{\prime}\right| \leq 1} S_{q^{\prime}-1} v^{j} \partial_{j} \Delta_{q^{\prime}} f_{q} \\
& =S_{q-1} v^{j} \partial_{j} f_{q}+\sum_{\left|q-q^{\prime}\right| \leq 1}\left(S_{q^{\prime}-1} v^{j}-S_{q-1} v^{j}\right) \partial_{j} \Delta_{q^{\prime}} f_{q} \\
& =S_{q-1} v^{j} \partial_{j} f_{q}+\Delta_{q-1} v^{j} \partial_{j} \Delta_{q+1} f_{q}-\Delta_{q-2} v^{j} \partial_{j} \Delta_{q-1} f_{q}
\end{aligned}
$$

Hence,

$$
\Delta_{q}(v \cdot \nabla f)=\sum_{\ell=1}^{3} R_{q}^{\ell}(v, f)+S_{q-1} v \cdot \nabla f_{q} .
$$

In the same way, we have

$$
\Delta_{q}\left(\operatorname{div}_{g}(G(v, f) f)\right)=\sum_{\ell=4}^{6} R_{q}^{\ell}(v, f)+\operatorname{div}_{g}\left(G\left(S_{q-1} v, S_{q-1} f\right) f_{q}\right) .
$$


Let us estimate the six terms appearing above. We have

Let us begin with $R_{q}^{1}(v, f)$. By definition of the paraproduct, we have

$$
R_{q}^{1}(v, f)=\sum_{j=1}^{d} \sum_{q^{\prime}} \Delta_{q}\left(S_{q^{\prime}-1} \partial_{j} f \Delta_{q^{\prime}} v^{j}\right) .
$$

As, if $\left|q-q^{\prime}\right|>2$ then the above term is equal to 0 , we deduce that

$$
\left\|H R_{q}^{1}(v(t), f(t))\right\|_{L^{p}\left(L^{2}\right)} \leq C \sum_{\left|q-q^{\prime}\right| \leq 2}\left\|H S_{q^{\prime}-1} \nabla f\right\|_{L^{\infty}\left(L^{2}\right)}\left\|\Delta_{q^{\prime}} v(t)\right\|_{L^{p}} .
$$

Using the fact that, if $\left|q-q^{\prime}\right| \leq 2$, then $\left\|H S_{q^{\prime}-1} \nabla f\right\|_{L^{\infty}\left(L^{2}\right)} \leq C 2^{q}\|H f(t)\|_{L^{\infty}\left(L^{2}\right)} \leq C 2^{q}$, we infer that

$$
\left\|H R_{q}^{1}(v(t), f(t))\right\|_{L^{p}\left(L^{2}\right)} \leq C 2^{q} \sum_{\left|q-q^{\prime}\right| \leq 2}\left\|\Delta_{q^{\prime}} v(t)\right\|_{L^{p}} \leq C \sum_{\left|q-q^{\prime}\right| \leq 2}\left\|\nabla \Delta_{q^{\prime}} v(t)\right\|_{L^{p}}
$$

Hence

$$
2^{q \sigma-\Phi_{q, \lambda}(t)}\left\|H R_{q}^{1}(v(t), f(t))\right\|_{L^{p}\left(L^{2}\right)} \leq C M_{\lambda}^{\sigma+1}(v) \sum_{\left|q-q^{\prime}\right| \leq 2} 2^{-\lambda \int_{0}^{t}\left\|S_{q} \nabla v\left(t^{\prime}\right)\right\|_{L^{\infty}} d t^{\prime}+\lambda \int_{0}^{t}\left\|S_{q^{\prime}} \nabla v\left(t^{\prime}\right)\right\|_{L^{\infty}} d t^{\prime}} .
$$

But, it is obvious that

$$
\int_{0}^{t}\left\|S_{q^{\prime}} \nabla v\left(t^{\prime}\right)\right\|_{L^{\infty}} d t^{\prime}-\int_{0}^{t}\left\|S_{q} \nabla v\left(t^{\prime}\right)\right\|_{L^{\infty}} d t^{\prime} \leq \int_{0}^{t}\left\|\left(S_{q^{\prime}}-S_{q}\right) \nabla v\left(t^{\prime}\right)\right\|_{L^{\infty}} d t^{\prime} .
$$

Using the fact that $\left|q-q^{\prime}\right| \leq 2$, we get

$$
\int_{0}^{t}\left\|S_{q^{\prime}} \nabla v\left(t^{\prime}\right)\right\|_{L^{\infty}} d t^{\prime}-\int_{0}^{t}\left\|S_{q} \nabla v\left(t^{\prime}\right)\right\|_{L^{\infty}} d t^{\prime} \leq C\left|q-q^{\prime}\right|\|\nabla v\|_{\widetilde{L}_{T}^{1}\left(C^{0}\right)} \cdot
$$

So it turns out that

$$
2^{q \sigma-\Phi_{q, \lambda}(t)}\left\|H R_{q}^{1}(v(t), f(t))\right\|_{L^{p}\left(L^{2}\right)} \leq 2^{C \lambda\|\nabla v\|_{\tilde{L}_{T}^{1}\left(C^{0}\right)}} M_{\lambda}^{\sigma+1}(v) .
$$

Now let us look at $R_{q}^{2}(v, f)$. By definition of the paraproduct, we have

$$
\begin{aligned}
R_{q}^{2}(v, f) & =-\sum_{j=1}^{d} \sum_{q^{\prime}}\left[S_{q^{\prime}-1} v^{j} \partial_{j} \Delta_{q^{\prime}}, \Delta_{q}\right] f \\
& =-\sum_{j=1}^{d} \sum_{q^{\prime}}\left[S_{q^{\prime}-1} v^{j}, \Delta_{q}\right] \partial_{j} \Delta_{q^{\prime}} f .
\end{aligned}
$$

The terms of the above sum are equal to 0 except if $\left|q-q^{\prime}\right| \leq 2$. Moreover, by definition of the operators $\Delta_{q}$, we have

$$
\left[S_{q^{\prime}-1} v^{j}, \Delta_{q}\right] \partial_{j} \Delta_{q^{\prime}} f(x)=2^{q d} \int_{\mathbb{R}^{d}} h\left(2^{q}(x-y)\right)\left(S_{q^{\prime}-1} v^{j}(x)-S_{q^{\prime}-1} v^{j}(y)\right) \partial_{j} \Delta_{q^{\prime}} f(y) d y .
$$

So we infer that

$$
\left\|H\left[S_{q^{\prime}-1} v^{j}, \Delta_{q}\right] \partial_{j} \Delta_{q^{\prime}} f(x)\right\|_{L^{2}(M)} \leq 2^{-q}\left\|\nabla S_{q^{\prime}-1} v\right\|_{L^{\infty}} 2^{q d}\left(\left(2^{q}|\cdot| \times\left|h\left(2^{q} \cdot\right)\right|\right) \star\left\|H \partial_{j} \Delta_{q^{\prime}} f\right\|_{L^{2}(M)}\right)(x) .
$$


Hence,

$$
\left\|H\left[S_{q^{\prime}-1} v^{j}, \Delta_{q}\right] \partial_{j} \Delta_{q^{\prime}} f(x)\right\|_{L^{p}\left(L^{2}(M)\right)} \leq 2^{-q}\left\|\nabla S_{q^{\prime}-1} v\right\|_{L^{\infty}}\left\|H \partial_{j} \Delta_{q^{\prime}} f\right\|_{L^{p}\left(L^{2}(M)\right)} .
$$

Then, we have, using Inequality (18),

$$
\begin{aligned}
& 2^{q \sigma-\Phi_{q, \lambda}(t)}\left\|H\left[S_{q^{\prime}-1} v^{j}, \Delta_{q}\right] \partial_{j} \Delta_{q^{\prime}} f\right\|_{L^{p}\left(L^{2}(M)\right)} \\
& \leq C M_{\lambda}^{\sigma}(f) \sum_{\left|q-q^{\prime}\right| \leq 2} 2^{C \lambda\|v\|_{\tilde{L}_{T}^{1}\left(C^{1}\right)}}\left(\left\|\nabla\left(S_{q^{\prime}-1}-S_{q}\right) v(t)\right\|_{L^{\infty}}+\left\|S_{q} v(t)\right\|_{L^{\infty}}\right) .
\end{aligned}
$$

So, we get

$$
\begin{aligned}
2^{q \sigma-\Phi_{q, \lambda}(t)} \| H R_{q}^{2}(v(t), f(t)) & \|_{L^{p}\left(L^{2}\right)} \leq C M_{\lambda}^{\sigma}(f) 2^{C \lambda\|v\|_{\tilde{L}^{1}\left(C^{1}\right)}} \\
& \times\left(\left\|S_{q} \nabla v(t)\right\|_{L^{\infty}}+\sum_{\left|q-q^{\prime}\right| \leq 2} \| \nabla\left(\Delta_{q^{\prime}} v(t) \|_{L^{\infty}}\right) .\right.
\end{aligned}
$$

For $R_{q}^{3}$, we have

$$
\begin{aligned}
\left\|H R_{q}^{3}(v, f)\right\|_{L^{p}\left(L^{2}\right)} & \leq C \sum_{\substack{\left|q^{\prime}-q^{\prime \prime}\right| \leq 1 \\
q^{\prime} \geq q-2}} 2^{q}\left\|\Delta_{q^{\prime}} v\right\|_{L^{p}}\left\|H \Delta_{q^{\prime \prime}} f\right\|_{L^{\infty}\left(L^{2}\right)} \\
& \leq C \sum_{q^{\prime} \geq q-2} 2^{q-q^{\prime}}\left\|\Delta_{q^{\prime}} \nabla v\right\|_{L^{p}}\|H f\|_{L^{\infty}\left(L^{2}\right)} .
\end{aligned}
$$

Hence,

$$
2^{q \sigma-\Phi_{q, \lambda}(t)}\left\|H R_{q}^{3}(v, f)\right\|_{L^{p}\left(L^{2}\right)} \leq C \sum_{q^{\prime} \geq q-2} 2^{(1+\sigma)\left(q-q^{\prime}\right)-\Phi_{q, \lambda}(t)+\Phi_{q^{\prime}, \lambda}(t)} M_{\lambda}^{\sigma+1}(v)\|H f\|_{L^{\infty}\left(L^{2}\right)} .
$$

Then, we see that the sum converges since

$$
\left|\Phi_{q, \lambda}(t)-\Phi_{q^{\prime}, \lambda}(t)\right| \leq \lambda\|\nabla v\|_{\widetilde{L}_{T}^{1}\left(C^{0}\right)}\left|q-q^{\prime}\right| \leq(\sigma-\beta)\left|q-q^{\prime}\right|
$$

and $1+\sigma-(\sigma-\beta)=1+\beta>0$. Hence, we get

$$
2^{q \sigma-\Phi_{q, \lambda}(t)}\left\|H R_{q}^{3}(v, f)\right\|_{L^{p}\left(L^{2}\right)} \leq C M_{\lambda}^{\sigma+1}(v)\|H f\|_{L^{\infty}\left(L^{2}\right)} .
$$

The estimate for $R_{q}^{4}(v, f)=R_{q}^{4,1}(v, f)+R_{q}^{4,2}(f)$ is the same as the estimate for $R_{q}^{1}(v, f)$. Indeed, we have

$$
\begin{aligned}
\left\|H R_{q}^{4,1}(v(t), f(t))\right\|_{L^{p}\left(L^{2}\right)} & \leq C \sum_{\left|q-q^{\prime}\right| \leq 2}\left\|\nabla_{g} H S_{q^{\prime}-1} f\right\|_{L^{\infty}\left(L^{2}\right)}\left\|\Delta_{q^{\prime}} \nabla v(t)\right\|_{L^{p}} \\
& \leq C \sum_{\left|q-q^{\prime}\right| \leq 2}\left\|\Delta_{q^{\prime}} \nabla v(t)\right\|_{L^{p}}
\end{aligned}
$$

where we used that $\left\|\nabla_{g} H S_{q^{\prime}-1} f\right\|_{L^{\infty}\left(L^{2}\right)} \leq C$. Hence, we conclude as for $R_{q}^{1}(v, f)$. Besides,

$$
\begin{aligned}
\left\|H R_{q}^{4,2}(f(t))\right\|_{L^{p}\left(L^{2}\right)} & \leq C \sum_{\left|q-q^{\prime}\right| \leq 2}\left\|\nabla_{g} H S_{q^{\prime}-1} f\right\|_{L^{\infty}\left(L^{2}\right)}\left\|\Delta_{q^{\prime}} \nabla_{g} U\right\|_{L^{p}} \\
& \leq C \sum_{\left|q-q^{\prime}\right| \leq 2}\left\|\Delta_{q^{\prime}} f(t)\right\|_{L^{p}}
\end{aligned}
$$


Hence, we conclude as for $R_{q}^{1}(v, f)$ and get

$$
2^{q \sigma-\Phi_{q, \lambda}(t)}\left\|H R_{q}^{5,2}(f(t))\right\|_{L^{p}\left(L^{2}\right)} \leq 2^{C \lambda\|\nabla v\|_{\widetilde{L}_{T}^{1}\left(C^{0}\right)}} M_{\lambda}^{\sigma}(f) .
$$

We write $R_{q}^{5}(v, f)=R_{q}^{5,1}(v, f)+R_{q}^{5,2}(f)$. The estimate for $R_{q}^{5}(v, f)$ is similar to the one for $R_{q}^{2}(v, f)$ with the only difference that we have to use the regularity of $\nabla v$. We have

$$
\left[\Delta_{q}, T_{\partial_{j} v^{i}}\right] f=-\sum_{j=1}^{d} \sum_{q^{\prime}}\left[S_{q^{\prime}-1} \partial_{j} v^{i}, \Delta_{q}\right] \partial_{j} \Delta_{q^{\prime}} f .
$$

The terms of the above sum are equal to 0 except if $\left|q-q^{\prime}\right| \leq 2$. Moreover, by definition of the operators $\Delta_{q}$, we have

$$
\left[S_{q^{\prime}-1} \partial_{j} v^{i}, \Delta_{q}\right] \Delta_{q^{\prime}} f(x)=2^{q d} \int_{\mathbb{R}^{d}} h\left(2^{q}(x-y)\right)\left(S_{q^{\prime}-1} \partial_{j} v^{i}(x)-S_{q^{\prime}-1} \partial_{j} v^{i}(y)\right) \Delta_{q^{\prime}} f(y) d y .
$$

So we infer that

$$
\left\|H R_{q}^{5,1}(v, f)\right\|_{L^{2}(M)} \leq 2^{-q}\left|\nabla^{2} S_{q^{\prime}-1} v\right| 2^{q d}\left(\left(2^{q}|\cdot| \times\left|h\left(2^{q} \cdot\right)\right|\right) \star\left\|\nabla_{g} H \Delta_{q^{\prime}} f\right\|_{L^{2}(M)}\right)(x) .
$$

Hence,

$$
\left\|H R_{q}^{5,1}(v, f)\right\|_{L^{p}\left(L^{2}(M)\right)} \leq 2^{-q}\left\|\nabla^{2} S_{q^{\prime}-1} v\right\|_{L^{p}}\left\|\nabla_{g} H \Delta_{q^{\prime}} f\right\|_{L^{\infty}\left(L^{2}(M)\right)} .
$$

Then, we have, using Inequality (18),

$$
\begin{aligned}
2^{q \sigma-\Phi_{q, \lambda}(t)}\left\|H R_{q}^{5,1}(v, f)\right\|_{L^{p}\left(L^{2}(M)\right)} & \leq C \sum_{\substack{\left|q-q^{\prime}\right| \leq 2 \\
q^{\prime \prime} \leq q^{\prime}-1}} 2^{(\sigma-1)\left(q-q^{\prime \prime}\right)-\Phi_{q, \lambda}(t)+\Phi_{q^{\prime \prime}, \lambda}(t)} M_{\lambda}^{\sigma+1}(v)\left\|\nabla_{g} H \Delta_{q^{\prime}} f\right\|_{L^{\infty}\left(L^{2}\right)} .
\end{aligned}
$$

Hence,

$$
2^{q \sigma-\Phi_{q, \lambda}(t)}\left\|H R_{q}^{5,1}(v, f)\right\|_{L^{p}\left(L^{2}(M)\right)} \leq C \sum_{q^{\prime \prime} \leq q+1} 2^{-\beta\left(q-q^{\prime \prime}\right)} M_{\lambda}^{\sigma+1}(v)\left\|\nabla_{g} H f\right\|_{L^{\infty}\left(L^{2}\right)}
$$

and the sum is uniformly bounded since $\sigma-1+\lambda\|\nabla v\|_{\widetilde{L}_{T}^{1}\left(C^{0}\right)} \leq-\beta$. Then, we argue in a similar way for $\left\|H R_{q}^{5,2}(f)\right\|_{L^{p}\left(L^{2}(M)\right)}$ and get

$$
\left\|H R_{q}^{5,2}(f)\right\|_{L^{p}\left(L^{2}(M)\right)} \leq 2^{-q}\left\|\nabla S_{q^{\prime}-1} f\right\|_{L^{p}\left(L^{2}(M)\right)}\left\|\nabla_{g} H \Delta_{q^{\prime}} f\right\|_{L^{\infty}\left(L^{2}(M)\right)} .
$$

and we conclude as above with $M_{\lambda}^{\sigma+1}(v)$ replaced by $M_{\lambda}^{\sigma}(f)$.

Finally, the estimate for $R_{q}^{6}(v, f)$ is exactly the same as the one for $R_{q}^{3}(v, f)$ since, we also have that $\left\|\nabla_{g} H f\right\|_{L^{\infty}\left(L^{2}\right)} \leq C$.

\section{Global existence}

Now, we turn to the proof of our main theorem. First, we notice that the local existence in with $v \in L_{l o c}^{\infty}\left([0, T) ; W^{1, r}\right) \cap L_{l o c}^{2}\left([0, T) ; W^{2, r}\right)$ and $f \in L_{l o c}^{\infty}\left([0, T) ; W^{1, r}\left(H^{-s}\right)\right)$ can be easily deduced from standard arguments. Moreover, from regularity estimates for the heat equation, we have for all $0<T_{0}<T, v \in L_{l o c}^{\infty}\left(\left(T_{0}, T\right) ; W^{2-\varepsilon, r}\right)$. 
We want to prove that we can extend the solution beyond the time $T$. It is enough to prove that $\nabla v \in L^{\infty}\left((0, T) \times \mathbb{R}^{2}\right)$.

The local existence result tells that, for any $T_{0}$ in $] 0, T[$, the solution $(v, f)$ of $(1)$ belongs to the space $L_{l o c}^{\infty}\left(\left[T_{0}, T\left[; W^{2-\varepsilon, r} \times W^{1, r}\left(H^{-s}\right)\right)\right.\right.$ for any $\varepsilon>0$. Sobolev type embeddings of Corollary 1.5 imply that

$$
(v, \tau) \in L_{l o c}^{\infty}\left(\left[T_{0}, T\left[; \widetilde{B}_{p, \infty}^{2-\varepsilon-2\left(\frac{1}{r}-\frac{1}{p}\right)} \times \widetilde{B}_{p, \infty}^{1-2\left(\frac{1}{r}-\frac{1}{p}\right)}\right)\right.\right.
$$

Choosing $\varepsilon<1-2 / r$ and $p=\infty$ in the above assertion implies that $(v, \tau) \in L_{\text {loc }}^{\infty}\left(\widetilde{C}^{1+\sigma} \times\right.$ $\left.\widetilde{C}^{\sigma}\left(H^{-s}\right)\right)$ where $\sigma=1-\varepsilon-2 / r>0$. So we can apply Theorem 1.7 and we can choose $T_{0}$ such that, with the notations of Theorem 2.1, we have

$$
\|\nabla v\|_{\widetilde{L}_{\left[T_{0}, T\right]}^{1}\left(C^{0}\right)} \leq \frac{\min (\sigma-\beta, 1-\sigma-\beta)}{3 \lambda} .
$$

The deteriorating regularity estimate of Theorem 2.1 applied with $\sigma$ and between $T_{0}$ and $T$ tells exactly that $f$ satisfies

$$
M_{\lambda}^{\sigma}(f) \leq 3\|f\|_{C^{\sigma}\left(H^{-s}\right)}+\frac{3 C}{\lambda} M_{\lambda}^{\sigma+1}(v) .
$$

Now, we have to estimate $\nabla v$. The two dimensional Navier-Stokes equation can be written as

$$
\partial_{t} v-\nu \Delta v=P(v \cdot \nabla v)+P D \tau
$$

where $P$ denotes the Leray projector on the divergence free vector field. Exactly along the same lines as in the proof of Theorem2.1, we have

$$
2^{q(\sigma+1)-\Phi_{q, \lambda}(t)}\left\|P(v \cdot \nabla v)-P\left(S_{q} v \cdot \nabla \Delta_{q} v\right)\right\|_{L^{\infty}} \leq C M_{\lambda}^{\sigma+1}(v)\left(\left\|S_{q} \nabla v(t)\right\|_{L^{\infty}}+\sum_{q^{\prime} \geq q} 2^{q-q^{\prime}}\left\|\nabla \Delta_{q^{\prime}} v(t)\right\|_{L^{\infty}}\right) .
$$

Moreover, it is obvious that

$$
2^{q\left(\sigma-\frac{1}{2}\right)-\Phi_{q, \lambda}(t)}\left\|P\left(S_{q} v \cdot \nabla \Delta_{q} v\right)\right\|_{L^{\infty}} \leq C\|v(t)\|_{H^{\frac{1}{2}}} M_{\lambda}^{\sigma+1}(v) .
$$

So it turns out that

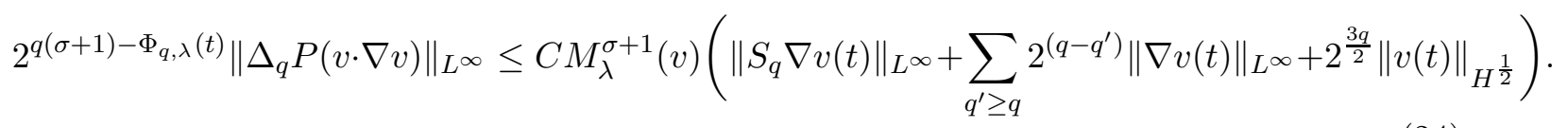

Using well known estimates on the heat equation (see for instance [4]) and Inequalities (23) and $(24)$, we get that

$$
M_{\lambda}^{\sigma+1}(v) \leq\left\|v_{0}\right\|_{C^{\sigma+1}}+\left(\frac{C}{\lambda}+2^{\frac{3 q}{2}} F_{q}\left(T_{0}, T\right)\right) M_{\lambda}^{s}(v)+\frac{C}{\nu} M_{\lambda}^{\sigma}(\tau)
$$

with

$$
F_{q}\left(T_{0}, T\right) \stackrel{\text { def }}{=} \sup _{t \in\left[T_{0}, T\right]} \int_{T_{0}}^{t} e^{c \nu 2^{2 q}\left(t-t^{\prime}\right)}\left\|v\left(t^{\prime}\right)\right\|_{H^{\frac{1}{2}}} d t^{\prime} .
$$

Hölder inequality implies immediately that

$$
F_{q}\left(T_{0}, T\right) \leq \frac{C}{\nu^{\frac{3}{4}}} 2^{-\frac{3 q}{2}}\|v\|_{L_{\left.T_{0}, T\right]}^{4}\left(H^{\frac{1}{2}}\right)}
$$


Moreover, it is easy to see that

$$
M_{\lambda}^{\sigma}(\tau) \leq M_{\lambda}^{\sigma}(f)
$$

So, we infer that

$$
M_{\lambda}^{\sigma+1}(v) \leq\left\|v_{0}\right\|_{C^{\sigma+1}}+\frac{3 C}{\nu}\left\|\tau_{0}\right\|_{C^{\sigma}}+\left(\frac{C}{\lambda}+\frac{C}{\lambda \nu}+\frac{C}{\nu^{\frac{3}{4}}}\|v\|_{L_{\left.T_{0}, T\right]}^{4}\left(H^{\frac{1}{2}}\right)}\right) M_{\lambda}^{\sigma+1}(v)
$$

Now it is enough to choose $T_{0}$ such that the quantity

$$
\left(\frac{C}{\lambda}+\frac{C}{\lambda \nu}+\frac{C}{\nu^{\frac{3}{4}}}\|v\|_{L_{\left.T_{0}, T\right]}^{4}\left(H^{\frac{1}{2}}\right)}\right)
$$

is small enough. Then as $\sigma$ is greater than 0 , the solution $(v, \tau)$ of the system $(1)$ is such that $(\nabla v, \tau)$ belongs to $L^{\infty}\left(\left[T_{0}, T\right] \times \mathbb{R}^{2}\right)$; this concludes the proof of Theorem 1.1.

\section{Acknowledgments}

The work of P.C. is partially supported by NSF-DMS grant 0504213. The work of N. M. is partially supported by NSF-DMS grant 0403983.

\section{References}

[1] H. Bahouri and J.-Y. Chemin. Équations de transport relatives á des champs de vecteurs non-lipschitziens et mécanique des fluides. Arch. Rational Mech. Anal., 127(2):159-181, 1994.

[2] R. B. Bird, C. Curtiss, R. Amstrong, and O. Hassager. Dynamics of polymeric liquids, Kinetic Theory Vol. 2,. Wiley, New York, 1987.

[3] J.-M. Bony. Calcul symbolique et propagation des singularités pour les équations aux dérivées partielles non linéaires. Ann. Sci. École Norm. Sup. (4), 14(2):209-246, 1981.

[4] J.-Y. Chemin. Théorèmes d'unicité pour le système de Navier-Stokes tridimensionnel. Journal d'Analyse Mathématique, 77(?):27-50, 1999.

[5] J.-Y. Chemin and N. Masmoudi. About lifespan of regular solutions of equations related to viscoelastic fluids. SIAM J. Math. Anal., 33(1):84-112 (electronic), 2001.

[6] P. Constantin, Nonlinear Fokker-Planck Navier-Stokes systems, Commun. Math. Sci. 3 (4), 531-544 (2005).

[7] P. Constantin, C. Fefferman, E. Titi and A. Zarnescu Regularity for coupled twodimensional nonlinear Fokker-Planck and Navier-Stokes systems Comm. Math. Phys (to appear)

[8] M. Doi, S.F. Edwards, The Theory of Polymer Dynamics, Oxford University Press, 1986.

[9] W. E, T. Li, and P. Zhang. Well-posedness for the dumbbell model of polymeric fluids. Comm. Math. Phys., 248(2):409-427, 2004.

[10] E. Fernández-Cara, F. Guillén, and R. R. Ortega. Some theoretical results for viscoplastic and dilatant fluids with variable density. Nonlinear Anal., 28(6):1079-1100, 1997. 
[11] E. Fernández-Cara, F. Guillén, and R. R. Ortega. Some theoretical results concerning non-Newtonian fluids of the Oldroyd kind. Ann. Scuola Norm. Sup. Pisa Cl. Sci. (4), 26(1):1-29, 1998.

[12] E. Fernández-Cara, F. Guillén, and R. R. Ortega. The mathematical analysis of viscoelastic fluids of the Oldryod kind. 2000.

[13] C. Guillopé and J.-C. Saut. Existence results for the flow of viscoelastic fluids with a differential constitutive law. Nonlinear Anal., 15(9):849-869, 1990.

[14] C. Guillopé and J.-C. Saut. Global existence and one-dimensional nonlinear stability of shearing motions of viscoelastic fluids of Oldroyd type. RAIRO Modél. Math. Anal. Numér., 24(3):369-401, 1990.

[15] B. Jourdain, T. Lelièvre, and C. Le Bris. Existence of solution for a micro-macro model of polymeric fluid: the FENE model. J. Funct. Anal., 209(1):162-193, 2004.

[16] F.-H. Lin, C. Liu, and P. Zhang. On hydrodynamics of viscoelastic fluids. Comm. Pure Appl. Math., 58(11):1437-1471, 2005.

[17] F.-H. Lin, C. Liu, and P. Zhang. On a Micro-Macro model for polymeric fluids near equilibrium Comm. Pure Appl. Math., (to appear)

[18] P.-L. Lions and N. Masmoudi. Global solutions for some Oldroyd models of nonNewtonian flows. Chinese Ann. Math. Ser. B, 21(2):131-146, 2000.

[19] F. Otto, A.E. Tzavaras, Continuity of velocity gradients in suspensions of rod-like molecules, SFB preprint Nr. 141, (2004).

[20] H. Zhang and P. Zhang. Local existence for the FENE-dumbbell model of polymeric fluids. Arch. Ration. Mech. Anal., 181(2):373-400, 2006. 